\title{
Genome characterization of bile-isolated Shewanella algae ACCC
}

\author{
Shu-Ying Tseng ${ }^{1}$, Kwong-Chung Tung ${ }^{1}$, Jan-Fang Cheng ${ }^{2}$, Yi-Hsuan Lee ${ }^{1}$, Zong-Yen Wu ${ }^{1}{ }^{1}$, , Yu-Kai Hong ${ }^{3}$, \\ Shi-Yu Chen ${ }^{3}$, Yao-Ting Huang ${ }^{3+}$ and Po-Yu Liu, ${ }^{4,5,6^{*+}}$ (D)
}

\begin{abstract}
Background: Shewanella algae has been recognized as an emerging human pathogen. However, not much is known about the mechanism of its pathogenesis and its adaptation to a special niche such as the hepatobiliary tract.

Results: In this study, we isolated the S. algae ACCC strain from human bile and performed whole genome sequencing. S. algae ACCC consists of a circular 4,743,354-bp chromosome with a GC content of 53.08\%, within 4080 protein coding sequences. The genome of strain ACCC contains a number of candidate genes which have been reported to be associated with bile adaption, including $h t p B$, exbBD, wecA, galU, adeFGH and phoPQ regulon.
\end{abstract}

Conclusions: Our results highlight the association of $S$. algae with a rare disease profile. Further studies are needed to shed light on the evolution of pathogenesis and the niche adaptation of S. algae.

Keywords: Shewanella, Cholecystitis, Whole genome sequencing, Colistin, Carbapenem

\section{Background}

Shewanella algae was first described in 1992 from various clinical samples in Japan [1]. Since then, the organism has been identified in aquatic ecosystems worldwide [2]. Studies have also suggested that it thrives in a wide-range of temperatures and sanities [3].

The manifestations of $S$. algae in human infections are protean; although sepsis, intra-abdominal infections, and soft tissue infections are present in the majority of cases [2]. The hepatobiliary tree is one of the most common sites of isolation and a major source of blood stream infection [2, 4]. Reports in Asia have further demonstrated hepatobiliary disease as an important risk factor for shewanellosis [4].

There are very few studies which have examined the pathogenicity of S. algae. Its ability for bile salt adaption is likely to be an important factor for its survival and subsequent infection of the hepatobiliary tract. Whole genome sequencing provides opportunities to address the issue
[5]. Currently, there are 12 genomes of S. algae accessible on NCBI databases (two clinical isolates from respiratory tract and soft tissue and ten environmental isolates). Here, we report the first whole genome sequence of bileisolated S. algae ACCC.

\section{Methods \\ Strain isolation and characterization}

The $S$. algae ACCC was isolated from a bile sample of a cholangitis patient and resistant to $5 \%$ ox-bile salts. The strain was identified as $S$. algae by the matrix-assisted laser desorption/ionization time-of-flight mass spectrometry (MALDI-TOF MS) technique, along with $16 \mathrm{~S}$ rRNA sequencing. The primers used for amplification of the 16S rRNA gene were B27F (5'-AGAGTTTGATCC TGGCTCAG-3') and U1492R (5'-GGTTACCTTGTT ACGACTT-3'). The PCR products were subsequently sequenced and compared with the 16S rRNA bacteria sequence database on the NCBI using BLASTn (optimized for megablast) search algorithm [6].

\footnotetext{
*Correspondence: pyliu@vghtc.gov.tw

${ }^{\dagger}$ Yao-Ting Huang and Po-Yu Liu contributed equally to this work

${ }^{6}$ Division of Infectious Diseases, Department of Internal Medicine,

Taichung Veterans General Hospital, Taichung 40705, Taiwan

Full list of author information is available at the end of the article
} 
Library preparation, whole-genome sequence archive, and de novo assembly

The bacterial genomic DNA was extracted from overnight culture of the $S$. algae ACCC using the QIAGEN Genomic-tip 100/G kit and Genomic DNA Buffer Set (QIAGEN, Valencia, CA) according to the manufacturer's instructions. Qubit dsDNA HS Assay kit and Qubit 2.0 fluorometer (Life Technologies) were used to measure DNA concentration. A total of $2 \mu \mathrm{g}$ of each DNA sample was used to build indexed PCR-free libraries, using a multiplexed high-throughput sequencing TruSeq DNA Sample Preparation Kit (Illumina, San Diego, CA) according to the manufacturer's protocols with minor modifications.

The genomic DNA was subjected to whole genome sequencing on an Illumina MiSeq sequencer using the paired-end $2 \times 250$ bp sequencing protocol and generated $2,927,608$ reads. The total read depth was 186 -fold coverage, with a mean read length of $301 \mathrm{bp}$. Read data was filtered using duk (http://duk.sourceforge.net/) $(\mathrm{ktrim}=\mathrm{r}$ $\mathrm{k}=23 \operatorname{mink}=11$ hdist $=1$ ). Low quality (Q-score $<10$ ) reads are trimmed and a read is retained if at least $50 \mathrm{bp}$ by FASTQX-toolkit (https://github.com/agordon/fastx _toolkit). Sequencing data were assembled using Velvet v. 1.2.07 and ALLPATHS v. R46652 using 31 bp $k$-mer size (https://github.com/agordon/fastx_toolkit). Sequencing data were assembled using Velvet v. 1.2.07 and ALLPATHS v. R46652.

\section{Annotation}

The annotation of the $S$. algae ACCC was performed using the National Center for Biotechnology Information (NCBI) Prokaryotic Genomes Automatic Annotation Pipeline (PGAAP), in which the prediction was carried out using Glimmer 3.02 [7]. The non-translated genes were predicted using tRNAScan-SE [8], RNAmmer [9], and RFAM [10]. Functional classification of the predicted genes was performed using RPSBLAST program v. 2.2.15 [11] in conjunction with the COGs (Clusters of Orthologous Groups of proteins) databases using an E-value threshold $<0.001$.

Identification and comparative analysis of virulence genes The candidate virulence genes in $S$. algae ACCC, MARS14, and C6G3 genomes were separately identified using the Virulence Factors Database (VFDB) [12]. The assembled genome is first aligned against VFDB protein sequences of the full dataset (Set B) using BLASTX under the following criteria: identity $>45 \%$, aligned length $>450 \mathrm{bp}$, alignment coverage $>95 \%$ and $E$-value $<1 \mathrm{e}-45$. If multiple virulence factor genes are overlapped at the same locus in the genome, only the best-aligned virulence factor gene is retained. The ACCCspecific virulence genes were further extracted by excluding those also found in MARS14 and C6G3. In addition, putative antibiotic resistance genes in ACCC strain and public available clinical S. algae genomes (MARS 14 and YHL) were predicted using ResFinder 3.0 [13].

\section{Phylogeny reconstruction}

We used OrhoANI to compute the ANI values [14], which has 254 citations, because whole-genome ANI now becomes the standard for species identification/ confirmation by NCBI during genome submission. The OrthoANI reconstructs the phylogeny in different ways. First, the genome is chopped into pieces of $1020 \mathrm{bp}$ and BLAST is used to compute the ANI of all homologous pairs. Finally, UPGMA is invoked to cluster the species using the ANI as distance metric. In order to further confirm the whole-genome phylogenetic accuracy of OrthoANI, we also reconstruct whole genome phylogeny independently using REALPHY [15], which is based on PhyML to infer the whole-genome phylogeny using maximum likelihood.

\section{Quality assurance}

Genomic DNA used for sequencing was purified from a pure culture taken from a single colony of the $S$. algae ACCC strain. The bacterium was identified as $S$. algae by both biochemical identification and the MALDI-TOF MS technique. The 16S rRNA gene was sequenced and BLAST was performed against the NCBI database, which revealed no potential contamination of the genomic library. The raw read data was filtered using duk, and quality trimmed with FASTQX-toolkit fastqTrimmer.

\section{Results and discussion}

\section{General Genome Features of S. algae ACCC}

The final assembled genome consisted of 74 scaffolds with a total size equal to $4,743,354 \mathrm{bp}$, with a mean $\mathrm{G}+\mathrm{C}$ content of 53.08\%. An illustration of the genomic contents in the genome of ACCC is shown in Fig. 1. The maximum contig size was equal to $589,495 \mathrm{bp}$, and the N50 size equal to $118,224 \mathrm{bp}$. The gene annotation included 4080 protein coding sequences (CDSs), 91 tRNA genes and 8 rRNA genes (Additional file 1: Table S1). The distribution of genes into COGs functional categories is shown in Additional file 2: Table S2. No extrachromosomal elements were detected in ACCC.

\section{Phylogenetic analysis}

Sequencing of the $16 \mathrm{~S}$ rRNA gene and a subsequent BLAST-search confirmed the taxonomic status of $S$. algae ACCC (Additional file 3: Figure S1). To further elucidate the phylogenetic relationships, whole genome 


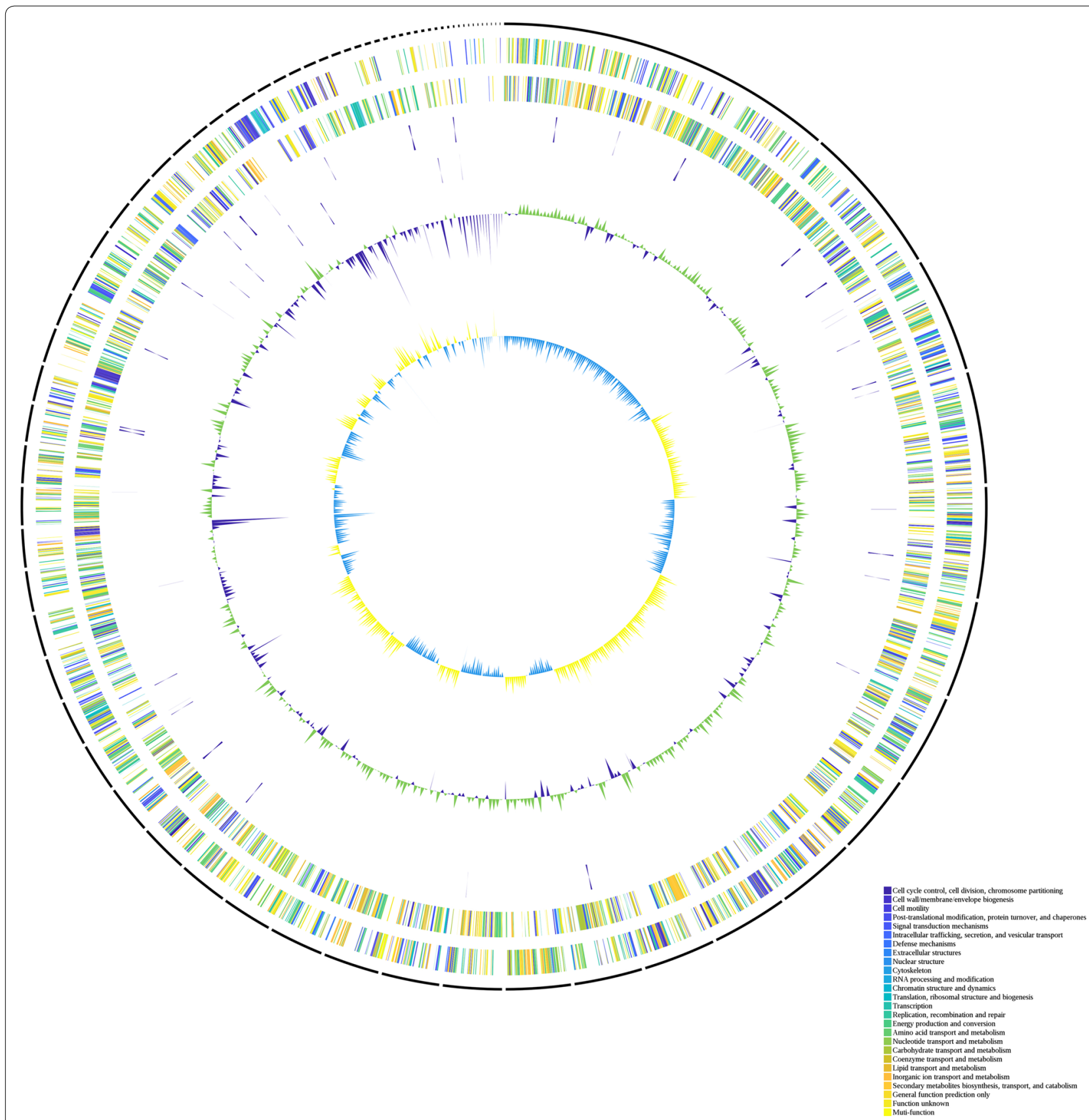

Fig. 1 Circular genome map of S. algae ACCC. Circles from the outside to inside showing: (1) DNA coordinates; $(2,3)$ function-based color coded mapping of the CDSs predicted on the forward and reverse strands. Functions are color-code; (4) tRNA genes; (5) rRNA genes; (6) GC plot showing regions above the average (green) and below (violet); (7) GC skew showing regions above average (yellow) and below (light blue)

DNA-sequence-based average nucleotide index analysis was performed and phylogenetic trees were constructed. The dendrogram illustrates that ACCC strain was most closely-related to C6G3 and MARS14, sharing an $\mathrm{ANI}>98 \%$ (Fig. 2). Independent analysis of Wholegenome phylogeny using REALPHY [15] also confirmed the phylogenetic position of ACCC is indeed most closely-related to C6G2 and MARS14 (Additional file 4: Figure S2).

\section{Analysis of virulence associated genes}

Further screening of the S. algae ACCC genome for putative virulence-associated genes was conducted by aligning ORF encoded protein sequences to the virulence 


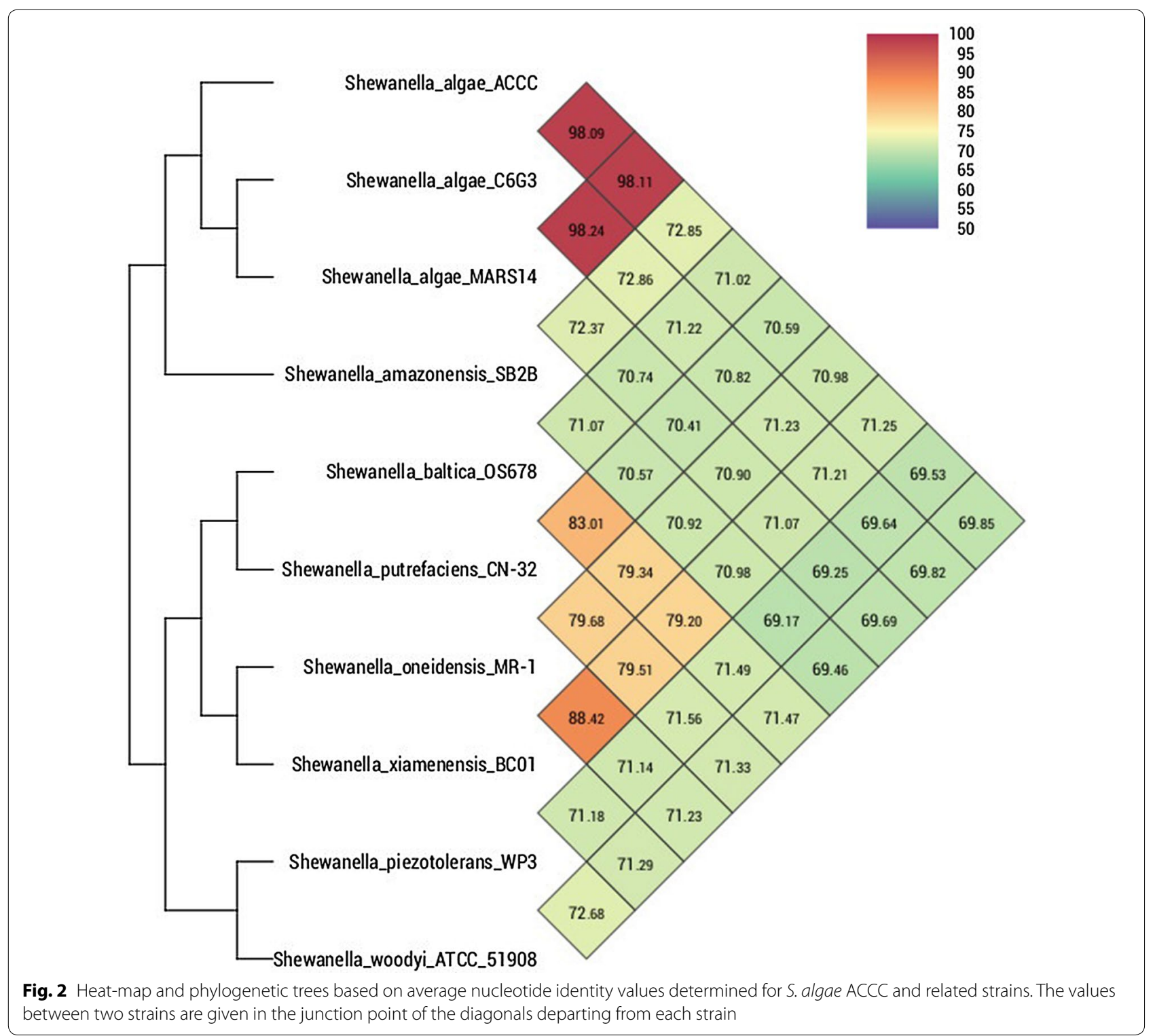

factor database. Comparative analysis of ACCC, clinical isolated MARS 14, and environmental isolated C6G2 revealed strain specific genes $m s h B$ which is associated with MSHA type IV pilus biogenesis. S. algae ACCC also contains conservative heat shock protein genes $\operatorname{clp} C$, $c l p E$, and $c l p P$. In addition, the histidine kinase gene cheA-2 was detected and the gene was demonstrated to be essential for chemotaxis [16]. We also predicted $b l a_{\mathrm{OXA}-55}$ in all tested genomes. The clinical significance of this finding warrants further investigation.

\section{Identification of genes related to bile stress}

Bile salts possess potent antimicrobial activity via damage membranes and DNA. To survive in bile and subsequently cause biliary tract infections, bacteria must have intrinsic resistance mechanisms to contend with bile stress [17]. Genomic analysis of $S$. algae ACCC showed the presence of numerous genes which may determine its bile resistance properties, supporting this strain's pathogenicity in causing cholecystitis. Comparative genomic analysis identified the presence of $h t p B$ in ACCC but not in clinical isolated MARS 14 . $h t p B$ encoding chaperonin, which has been reported to implicate in bacteria response to bile [18]. We also predicted conservative genes associated with bile adaption. S. algae ACCC possessed exbBD encoding Ton energy transduction system implicated in the response to bile [19]. We also detected phoPQ regulon, galU, and wecA 
involved in bile resistance [17]. The gene encoding bileinducible molecular chaperone DnaK was also identified [17]. The resistance-nodulation-cell division family members have been associated with tolerance to bile salts, multidrug resistance and biofilm formation [20]. Analysis of the genome of strain ACCC revealed the existence of genes encoding resistance-nodulation-cell division pump AdeFGH. Further studies are required to verify its genetic properties, along with the virulence potential, evolution traits for its zoonotic properties and spreading capabilities.

\section{Additional files}

Additional file 1: Table S1. General features of S. algae ACCC genome.

Additional file 2: Table S2. COG functional categories of S. algae ACCC genome.

Additional file 3: Figure S1. Phylogenetic tree based on 165 rRNA gene sequences showing the phylogenetic position of Shewanella algae ACCC.

Additional file 4: Figure S2. Phylogenetic tree based on whole-genome sequences showing the phylogenetic position of Shewanella algae ACCC.

\section{Abbreviations}

ANI: average nucleotide index; MALDI-TOF: matrix-assisted laser desorption/ ionization time-of-flight mass spectrometry.

\section{Authors' contributions}

JC, YH and PL proposed this work. ST, KT, ZW collected samples, performed experiments. SC, YH, PL analyzed data, wrote first draft of manuscript. YH, $\mathrm{PL}, \mathrm{KT}$ reviewed and edited manuscript. YH and PL have critically edited the revised version of manuscript. All authors read and approved the final manuscript.

\section{Author details \\ 1 Department of Veterinary Medicine, National Chung Hsing University, Taichung 40227, Taiwan. ${ }^{2}$ Department of Energy, Joint Genome Institute, Walnut Creek, CA 94598, USA. ${ }^{3}$ Department of Computer Science and Infor- mation Engineering, National Chung Cheng University, Chia-Yi 62102, Taiwan. ${ }^{4}$ Department of Nursing, Shu-Zen Junior College of Medicine and Manage- ment, Kaohsiung 82144, Taiwan. ${ }^{5}$ Rong Hsing Research Center for Transla- tional Medicine, National Chung Hsing University, Taichung 40227, Taiwan. \\ ${ }^{6}$ Division of Infectious Diseases, Department of Internal Medicine, Taichung Veterans General Hospital, Taichung 40705, Taiwan.}

\section{Acknowledgements}

We would like to take this opportunity to thank Dr. Lee Pan for his support in Kyoto and Osaka.

\section{Competing interests}

The authors declare that they have no competing interests.

\section{Availability of data and materials}

The genome sequence of Shewanella algae ACCC has been deposited in DDBJ/EMBL/GenBank under Project Accession Number LVCY00000000. The version described in work is version LVCY00000000.1.

\section{Consent for publication}

Not applicable.

Ethics approval and consent to participate

Not applicable.

\section{Funding}

We are grateful to Taiwan's Ministry of Science and Technology (MOST) and Taichung Veterans General Hospital for funding. YTH was supported in part by a Grant (106-2221-E-194-056-MY3) from Taiwan's Ministry of Science and Technology (MOST). PYL was supported by Grant (TCVGH-1073901B) from Taichung Veterans General Hospital.

\section{Publisher's Note}

Springer Nature remains neutral with regard to jurisdictional claims in published maps and institutional affiliations.

Received: 30 June 2018 Accepted: 12 September 2018

Published online: 18 September 2018

\section{References}

1. Nozue H, Hayashi T, Hashimoto Y, Ezaki T, Hamasaki K, Ohwada K, Terawaki Y. Isolation and characterization of Shewanella alga from human clinical specimens and emendation of the description of S. alga Simidu et al., 1990, 335. Int J Syst Bacteriol. 1992:42(4):628-34.

2. Janda JM, Abbott SL. The genus Shewanella: from the briny depths below to human pathogen. Crit Rev Microbiol. 2014;40(4):293-312.

3. Holt HM, Gahrn-Hansen B, Bruun B. Shewanella algae and Shewanella putrefaciens: clinical and microbiological characteristics. Clin Microbiol Infect. 2005;11(5):347-52.

4. Liu PY, Lin CF, Tung KC, Shyu CL, Wu MJ, Liu JW, Chang CS, Chan KW, Huang JA, Shi ZY. Clinical and microbiological features of shewanella bacteremia in patients with hepatobiliary disease. Intern Med. 2013;52(4):431-8.

5. Ahmed N. A flood of microbial genomes-do we need more? PLoS ONE. 2009:4(6):e5831.

6. Camacho C, Coulouris G, Avagyan V, Ma N, Papadopoulos J, Bealer K, Madden TL. BLAST+: architecture and applications. BMC Bioinform. 2009:10:421.

7. Delcher AL, Bratke KA, Powers EC, Salzberg SL. Identifying bacterial genes and endosymbiont DNA with glimmer. Bioinformatics. 2007;23(6):673-9.

8. Lowe TM, Eddy SR. tRNAscan-SE: a program for improved detection of transfer RNA genes in genomic sequence. Nucleic Acids Res. 1997:25(5):955-64.

9. Lagesen K, Hallin P, Rodland EA, Staerfeldt HH, Rognes T, Ussery DW. RNAmmer: consistent and rapid annotation of ribosomal RNA genes. Nucleic Acids Res. 2007;35(9):3100-8.

10. Griffiths-Jones S, Moxon S, Marshall M, Khanna A, Eddy SR, Bateman A. Rfam: annotating non-coding RNAs in complete genomes. Nucleic Acids Res. 2005;33(Database issue):D121-4.

11. Altschul SF, Gish W, Miller W, Myers EW, Lipman DJ. Basic local alignment search tool. J Mol Biol. 1990;215(3):403-10.

12. Chen L, Zheng D, Liu B, Yang J, Jin Q. VFDB 2016: hierarchical and refined dataset for big data analysis - 10 years on. Nucleic Acids Res. 2016:44(D1):D694-7.

13. Zankari E, Hasman H, Cosentino S, Vestergaard M, Rasmussen S, Lund $\mathrm{O}$, Aarestrup FM, Larsen MV. Identification of acquired antimicrobial resistance genes. J Antimicrob Chemother. 2012;67(11):2640-4.

14. Lee I, Ouk Kim Y, Park SC, Chun J. OrthoANI: an improved algorithm and software for calculating average nucleotide identity. Int I Syst Evol Microbiol. 2016;66(2):1100-3.

15. Bertels F, Silander OK, Pachkov M, Rainey PB, van Nimwegen E. Automated reconstruction of whole-genome phylogenies from shortsequence reads. Mol Biol Evol. 2014;31(5):1077-88.

16. Gosink KK, Kobayashi R, Kawagishi I, Hase CC. Analyses of the roles of the three cheA homologs in chemotaxis of Vibrio cholerae. J Bacteriol. 2002;184(6):1767-71.

17. Begley M, Gahan CG, Hill C. The interaction between bacteria and bile. FEMS Microbiol Rev. 2005;29(4):625-51.

18. Okoli AS, Wadstrom T, Mendz GL. MiniReview: bioinformatic study of bile responses in Campylobacterales. FEMS Immunol Med Microbiol. 2007:49(1):101-23. 
19. Hamner S, McInnerney K, Williamson K, Franklin MJ, Ford TE. Bile salts affect expression of Escherichia coli O157:H7 genes for virulence and iron acquisition, and promote growth under iron limiting conditions. PLOS ONE. 2013;8(9):e74647.

20. Lopez M, Blasco L, Gato E, Perez A, Fernandez-Garcia L, Martinez-Martinez L, Fernandez-Cuenca F, Rodriguez-Bano J, Pascual A, Bou G, et al.
Response to bile salts in clinical strains of Acinetobacter baumannii lacking the AdeABC efflux pump: virulence associated with quorum sensing. Front Cell Infect Microbiol. 2017:7:143.
Ready to submit your research? Choose BMC and benefit from:

- fast, convenient online submission

- thorough peer review by experienced researchers in your field

- rapid publication on acceptance

- support for research data, including large and complex data types

- gold Open Access which fosters wider collaboration and increased citations

- maximum visibility for your research: over 100M website views per year

At BMC, research is always in progress.

Learn more biomedcentral.com/submissions 